\title{
Fractional Fuzzy Adaptive Sliding-Mode Control of a 2-DOF Direct-Drive Robot Arm
}

\author{
Mehmet Önder Efe, Senior Member, IEEE
}

\begin{abstract}
This paper presents a novel parameter adjustment scheme to improve the robustness of fuzzy sliding-mode control achieved by the use of an adaptive neuro-fuzzy inference system (ANFIS) architecture. The proposed scheme utilizes fractionalorder integration in the parameter tuning stage. The controller parameters are tuned such that the system under control is driven toward the sliding regime in the traditional sense. After a comparison with the classical integer-order counterpart, it is seen that the control system with the proposed adaptation scheme displays better tracking performance, and a very high degree of robustness and insensitivity to disturbances are observed. The claims are justified through some simulations utilizing the dynamic model of a 2-DOF direct-drive robot arm. Overall, the contribution of this paper is to demonstrate that the response of the system under control is significantly better for the fractional-order integration exploited in the parameter adaptation stage than that for the classical integer-order integration.
\end{abstract}

Index Terms-Adaptive fuzzy control, fractional order control, sliding mode control.

\section{INTRODUCTION}

$\mathbf{T}$ HE NOTION of adaptiveness has been a core issue in many instances in feedback control. Many approaches have been considered for obtaining a better response from a closed-loop system that is equipped with a parameter tuning mechanism. Model reference adaptive control (MRAC) and self-tuning control (STC) are the examples that are now considered in the textbooks of adaptive control [1]. In its essence, the need for adaptation is related either to the changing process parameters or to the uncertainties. While the variants of MRAC and STC are proposed, the discovery of error backpropagation stimulated the researchers and engineers who have reported successful applications of online learning neural controllers (see [2]) and adaptive fuzzy control schemes [3]. The latter have become particularly popular as they exploit the verbal descriptions and verbal quantifications of the physical phenomena in a hierarchically rule-based structure [4]. Given a task to be accomplished, the process describing the best evolution of the adjustable parameters is the process of

Manuscript received October 25, 2007; revised February 26, 2008 and May 9, 2008. Current version published November 20, 2008. This work was supported by Turkish Scientific Council (TÜBITAK) Contract 107E137. This paper was recommended by Associate Editor S. Yang.

The author is with the Department of Electrical and Electronics Engineering, TOBB Economics and Technology University, 06560 Ankara, Turkey (e-mail: onderefe@etu.edu.tr).

Color versions of one or more of the figures in this paper are available online at http://ieeexplore.ieee.org.

Digital Object Identifier 10.1109/TSMCB.2008.928227 learning, which is sometimes called adaptation, tuning, adjustment, or optimization, all referring to the same reality in the context of fuzzy control. Many approaches have been proposed, namely, gradient descent, Levenberg-Marquardt technique, conjugate gradient method, Lyapunov-based techniques are just to name a few; a good treatment can be found in [4] and [5].

A successful application of fuzzy information processing is the realm of fuzzy sliding-mode control (FSMC). Numerous important results have been reported. In [6] and [7], fuzzy logic is used to obtain an adaptive boundary layer, and it is shown that the proposed method successfully alleviates the difficulties caused by the unmodeled dynamics. Palm [6] presents the extension of the boundary layer design to systems of order greater than two. Erbatur et al. [8] consider the robot dynamics studied in this paper and utilize the concept of fuzziness for reducing the adverse effects of chattering. In [9], several control points are chosen on the sliding line, and optimal moves of these points are designed via linguistic descriptions of fuzzy logic ending up with a successful emergence of the sliding regime. In [10], based on the limited state knowledge, two adaptive fuzzy models to obtain the functions embodying the system dynamics are developed, and the obtained functions have been utilized in sliding-mode control of a multiinput-multi-output plant. The adaptation of the reaching law parameter is proposed in [11], where a quicker reaching with suppressed oscillations in the response of a flexible robot is demonstrated with a comparison with classical sliding-mode controller. Kaynak et al. [12] present a thorough survey on computationally intelligent systems improving the performance of sliding-mode controllers. More recently, the FSMC framework has been applied to estimate the nonlinear terms in the dynamics of a robot [13], to synchronize two chaotic gyros [14], and to control a nonaffine system, a pneumatic valve in [15].

A common feature of all these methods and the cited research is the fact that the differentiation and integration, or shortly differintegration, of quantities are performed in integer order, i.e., $\mathbf{D}:=d / d t$ for the differentiation with respect to $t$ and $\mathbf{I}=\mathbf{D}^{-1}$ for the integration over $t$ in the traditional sense. A significantly different branch of mathematics, called fractional calculus, suggests operators $\mathbf{D}^{\beta}$ 's with $\beta \in \Re$, [16], [17], and it becomes possible to write $\mathbf{D} f=\mathbf{D}^{1 / 2}\left(\mathbf{D}^{1 / 2} f\right)$. Expectedly, Laplace and Fourier transforms in fractional calculus are available to exploit in closed-loop control system design, involved with $s^{\beta}$ or $(j \omega)^{\beta}$ generic terms, respectively.

Fractional calculus and dynamics described by fractional differential equations (FDEs) are becoming more and more 
popular as the underlying facts about the differentiation and integration are significantly different from the integer-order counterparts, and beyond this, many real-life systems are described better by FDEs, e.g., heat equation, telegraph equation, and a lossy electric transmission line are all involved with fractionalorder differintegration operators. A majority of works published so far have concentrated on the fractional variants of the PID controller, which has fractional-order differentiation and fractional-order integration, implemented for the control of linear dynamic systems, for which the issues of parameter selection, tuning, stability, and performance are rather mature concepts utilizing the results from complex analysis and frequency domain methods of control theory (see [18]) than those involving the nonlinear models (see [19]) and parameter changes in the approaches.

Parameter tuning in adaptive control systems is a central part of the overall mechanism alleviating the difficulties associated with the changes in the parameters and uncertainties that influence the closed-loop performance. Many remarkable studies are reported in the past, and the field of adaptation has become a blend of techniques of dynamical systems theory, optimization, heuristics (intelligence), and soft computing. Today, due to the advent of very high speed computers and networked computing facilities, even within microprocessorbased systems, tuning of system parameters based upon some set of observations and decisions has greatly been facilitated. In [1], an in-depth discussion for parameter tuning in continuous and discrete time is presented. Particularly for gradient descent rule for MRAC, which is considered in the integer order in [1], it has been implemented in fractional order by Vinagre et al. [20], where the integer-order integration is replaced with an integration of fractional order of 1.25 , and by Ladaci and Charef [21], where the good performance in noise rejection is emphasized.

In the related literature, the absence of methods designed and implemented via fractional differintegration in robust and nonlinear control is visible. The purpose of this paper is to fill this gap to the extent that covers the following: 1) better robustness and noise rejection capabilities than those utilizing traditional integer-order operators; 2) better tracking capability and better system response; 3) conditions for hitting in finite time; and 4) sliding-mode control based on fractionalorder adaptation. The aforementioned features constitute the major results and contributions of the paper advancing the subject area to the fractional-order operator-based adaptation schemes.

This paper is organized as follows. In the next section, we briefly consider the conventional sliding-mode control for multi-input-multi-output systems. Section III is devoted to the adaptive neuro-fuzzy inference system (ANFIS) structure and the relation between its inputs and output. The supervised training of the ANFIS structure with fractional-order adaptation scheme is presented in Section IV. The conditions for the sign equivalence between the control error and the switching function are given in Section $\mathrm{V}$, where the supervisory information is extracted from the available quantities. The dynamics of the plant is described in Section VI, and simulation results and the concluding remarks are given at the end of this paper.

\section{Overview OF SLiding-Mode Control}

Consider a general dynamic system described by

$\theta_{i}^{\left(r_{i}\right)}=f_{i}(\boldsymbol{\Theta})+\tilde{f}_{i}(\boldsymbol{\Theta})+\sum_{j=1}^{m}\left(g_{i j}(\boldsymbol{\Theta})+\tilde{g}_{i j}(\boldsymbol{\Theta})\right) \tau_{j}$,
$i=1,2, \ldots, n$

where $\boldsymbol{\Theta}=\left(\theta_{1}, \dot{\theta}_{1}, \ldots, \theta_{1}^{\left(r_{1}-1\right)}, \theta_{2}, \quad \dot{\theta}_{2}, \ldots, \theta_{2}^{\left(r_{2}-1\right)}, \ldots, \theta_{n}\right.$, $\left.\dot{\theta}_{n}, \ldots, \theta_{n}^{\left(r_{n}-1\right)}\right)^{\mathrm{T}}$ is the state vector of the entire system, $r_{i}$ is the order of the $i$ th subsystem, $f_{i}(\mathbf{\Theta})$ and $g_{i j}(\mathbf{\Theta})$ are scalar functions of the state vector describing the nominal (known) part of the dynamics, $\tilde{f}_{i}(\boldsymbol{\Theta})$ and $\tilde{g}_{i j}(\boldsymbol{\Theta})$ are the bounded uncertainties on these functions, and the input vector $\mathbf{T}=$ $\left(\tau_{1}, \tau_{2}, \ldots, \tau_{n}\right)^{\mathrm{T}}$ is the manipulated variable. This system of equations can be rewritten compactly as

$$
\dot{\boldsymbol{\Theta}}=F(\boldsymbol{\Theta})+\tilde{F}(\boldsymbol{\Theta})+(G(\boldsymbol{\Theta})+\tilde{G}(\boldsymbol{\Theta})) \mathbf{T}
$$

where $F(\boldsymbol{\Theta})$ and $\tilde{F}(\boldsymbol{\Theta})$ are $\sum_{i=1}^{n} r_{i} \times 1$ dimensional vectors, and $G(\boldsymbol{\Theta})$ and $\tilde{G}(\boldsymbol{\Theta})$ are $\sum_{i=1}^{n} r_{i} \times n$ dimensional matrices. The designer has the nominal plant dynamics given by $\dot{\Theta}=$ $F(\boldsymbol{\Theta})+G(\boldsymbol{\Theta}) \mathbf{T}$.

Standard approach for the design of a sliding-mode controller entails a switching function defined as

$$
\begin{aligned}
\mathbf{s} & =\left(s_{1}, s_{2}, \ldots, s_{n}\right)^{\mathrm{T}} \\
& =\boldsymbol{\Lambda}\left(\boldsymbol{\Theta}-\boldsymbol{\Theta}_{d}\right)
\end{aligned}
$$

where $\boldsymbol{\Theta}_{d}=\left(\theta_{d, 1}, \dot{\theta}_{d, 1}, \ldots, \theta_{d, 1}^{\left(r_{1}-1\right)}, \theta_{d, 2}, \dot{\theta}_{d, 2} \quad \ldots, \theta_{d, 2}^{\left(r_{2}-1\right)}\right.$, $\left.\ldots, \theta_{d, n}, \dot{\theta}_{d, 2}, \ldots, \theta_{d, n}^{\left(r_{n}-1\right)}\right)^{\mathrm{T}}$ is the vector of desired states, and the locus described by $\mathbf{s}=\mathbf{0}$ corresponds to the sliding manifold or the switching hypersurface. The entries of $\Lambda$ are chosen such that the $i$ th component of the switching manifold has the structure

$$
s_{i}=\left(\frac{d}{d t}+\lambda_{i}\right)^{r_{i}-1}\left(\theta_{i}-\theta_{d, i}\right), \quad i=1,2, \ldots, n
$$

where $\lambda_{i}>0$. Choosing a Lyapunov function candidate as in (5) and setting the control vector as given in (6), one gets the equality in (7), provided that the inverse $(\boldsymbol{\Lambda} G(\boldsymbol{\Theta}))^{-1}$ exists

$$
\begin{aligned}
V= & \frac{1}{2} \mathbf{s}^{\mathrm{T}} \mathbf{s} \\
\boldsymbol{\tau}_{\mathbf{S M C}}= & -(\boldsymbol{\Lambda} G(\boldsymbol{\Theta}))^{-1} \boldsymbol{\Lambda}\left(F(\boldsymbol{\Theta})-\dot{\boldsymbol{\Theta}}_{d}\right) \\
& -(\boldsymbol{\Lambda} G(\boldsymbol{\Theta}))^{-1} \mathbf{Q} \operatorname{sgn}(\mathbf{s}) \\
\dot{\mathbf{s}}= & -\mathbf{P Q} \operatorname{sgn}(\mathbf{s})+(\mathbf{P}-\mathbf{I}) \boldsymbol{\Lambda}\left(\dot{\boldsymbol{\Theta}}_{d}-F(\boldsymbol{\Theta})\right)+\boldsymbol{\Lambda} \tilde{F}
\end{aligned}
$$

where $\mathbf{P}:=\boldsymbol{\Lambda}(G+\tilde{G})(\boldsymbol{\Lambda} G)^{-1}$, which is very close to the identity matrix, and $\mathbf{Q}$ is a positive definite diagonal matrix chosen by the designer. If one sets $\mathbf{T}:=\boldsymbol{\tau}_{\mathrm{SMC}}$, then the system enters the sliding mode after a reaching phase.

The expression in (7) can be interpreted as follows:

1) If there are no uncertainties, i.e., $\tilde{F}=\mathbf{0}$ and $\tilde{G}=\mathbf{0}$, then we have $\dot{\mathbf{s}}=-\mathbf{Q} \operatorname{sgn}(\mathbf{s})$, and $\mathbf{s}^{\mathrm{T}} \dot{\mathbf{s}}<0$ is satisfied with any positive definite $\mathbf{Q}$. In this case, we have $\mathbf{P}=\mathbf{I}$, and this result is straightforward. 
2) If only $\tilde{G}=\mathbf{0}$, we obtain $\dot{\mathbf{s}}=-\mathbf{Q} \operatorname{sgn}(\mathbf{s})+\boldsymbol{\Lambda} \tilde{F}$, and $\mathbf{s}^{\mathrm{T}} \dot{\mathbf{s}}<0$ is satisfied if $\mathbf{Q}$ is a positive definite diagonal matrix and the $i$ th entry in the diagonal of $\mathbf{Q}$ is greater than the supremum value of the $i$ th row of $|\boldsymbol{\Lambda} \tilde{F}|$. This would preserve the sign of $\mathbf{s}$ in the presence of the term $\boldsymbol{\Lambda} \tilde{F}$, and the numerical computation would require the bounds of the uncertainties. In this case, we have $\mathbf{P}=\mathbf{I}$ too.

3) In the most general case, where neither of $\tilde{F}$ nor $\tilde{G}$ is zero, the expression in (7) is obtained. In this case, depending on the uncertainties influencing the input gains $(\tilde{G}$ 's), the matrix $\mathbf{P}$ is very close to the identity matrix, and utilizing the uncertainty bounds, the matrix $\mathbf{Q}$ can be chosen such that the sign of $\mathbf{s}$ is preserved and $\mathbf{s}^{\mathrm{T}} \dot{\mathbf{s}}<0$ is satisfied.

With an appropriate choice of $\mathbf{Q}, \mathbf{s}^{\mathrm{T}} \dot{\mathbf{s}}<0$ can be obtained for $\|\mathbf{s}\|>0$, and this result indicates that the error vector defined by the difference $\boldsymbol{\Theta}-\boldsymbol{\Theta}_{d}$ is attracted by the subspace characterized by $\mathbf{s}=\mathbf{0}$ and moves toward the origin according to what is prescribed by $\mathbf{s}=\mathbf{0}$. The motion during $\mathbf{s} \neq \mathbf{0}$ is called the reaching mode, whereas the motion when $\mathbf{s}=\mathbf{0}$ is called the sliding mode. During the latter dynamic mode, the closed-loop system exhibits certain degrees of robustness against the modeling uncertainties, yet the system is sensitive to noise as the sign of a quantity that is very close to zero determines the control action heavily.

It is straightforward to show that a hitting to $s_{i}=0$ occurs, and the hitting time $\left(t_{h, i}\right)$ for the $i$ th subsystem satisfies the inequality $t_{h, i} \leq\left(\left|s_{i}(0)\right| / \mathbf{Q}_{i i}\right)$. One can refer to [22]-[24] for an in-depth discussion on sliding-mode control. Our goal will be to obtain the sliding regime by utilizing an ANFIS structure introduced next.

\section{ANFIS}

Fuzzy logic offers one natural way for representing knowledge that is similar to human reasoning. Partitioning the input space by the use of fuzzy membership functions, determining the local conclusions through rules, and utilizing a flexible method of combining the localized information result in a highly interpretable and useful model that acts globally. ANFIS, in this respect, is one of the widely known architectures exploiting the power of connectionist structures while maintaining the verbal nature through membership functions and inference mechanisms [25], [26].

In Fig. 1, the general structure of a fuzzy inference system is shown. The crisp inputs are fuzzified through the computation of membership functions. This practically maps the input space to a feature space characterized by fuzzy sets. In the inference engine, computed membership values for each rule

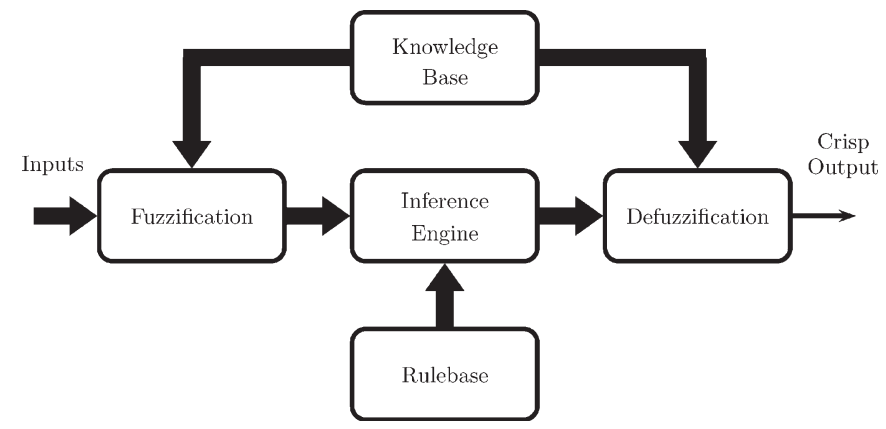

Fig. 1. Structure of a fuzzy inference system.

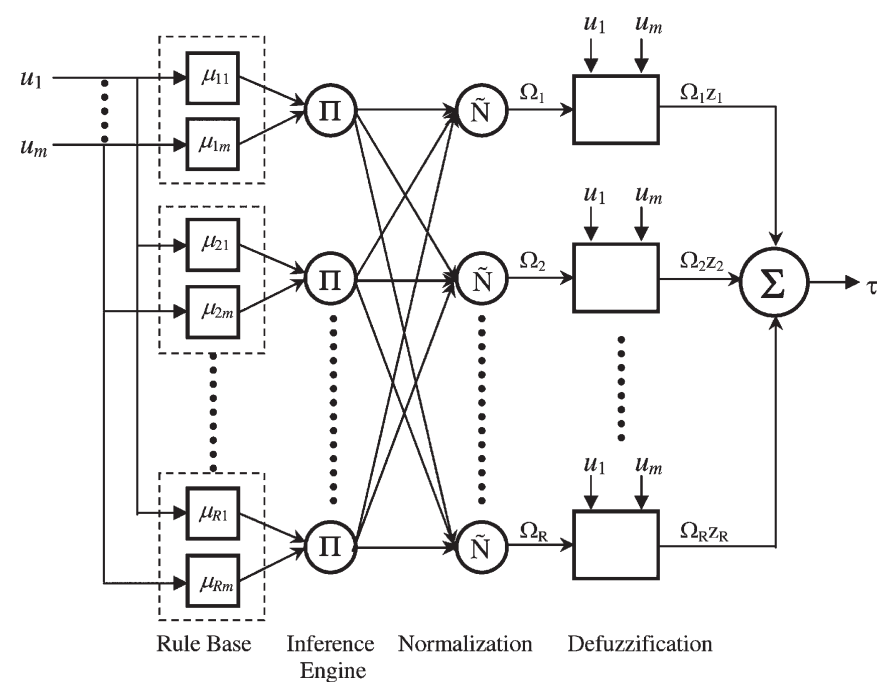

Fig. 2. Internal structure of ANFIS.

are converted into a firing strength that indicates the activation level of the rule. The parameters of the membership functions and auxiliary parameters are stored in the knowledge base, and a defuzzifier maps the feature vector to a scalar output value, which is crisp.

In Fig. 2, the internal structure of ANFIS is shown. As shown also in the figure, defining $w_{i}$ and $\Omega_{i}$ as the firing strength of the $i$ th rule and the normalized firing strength of the $i$ th rule, respectively, the input-output relation of the ANFIS structure with the product inference, the first-order Sugeno-type defuzzifier, and the rule-base structure containing $R$ rules as presented hereafter is described compactly in (8a)-(8d) (see [27]). Note that $\mathbb{U}_{i}, \mathbb{V}_{i}$, and $\mathbb{W}_{i}$ stand for the fuzzy sets characterized by the membership functions, that $y_{i}$ in the $i$ th rule is the local conclusion suggested by the rule, and that $n_{1}, n_{2}$, and $n_{m}$ correspond to the number of linguistic labels for the first, second, and $m$ th input variables, respectively (see the equation found at the bottom of the page).

$\begin{array}{cccccccccccccccc}\text { IF } & u_{1} & \text { is } & \mathbb{U}_{1} & \text { AND } & u_{2} & \text { is } & \mathbb{V}_{1} & \text { AND } & \cdots & \text { AND } & u_{m} & \text { is } & \mathbb{W}_{1} & \text { THEN } & y_{1}=z_{1} \\ \text { IF } & u_{1} & \text { is } & \mathbb{U}_{1} & \text { AND } & u_{2} & \text { is } & \mathbb{V}_{1} & \text { AND } & \cdots & \text { AND } & u_{m} & \text { is } & \mathbb{W}_{2} & \text { THEN } & y_{2}=z_{2} \\ & & & \vdots & & & & & & & \vdots & & & & & \\ \text { IF } & u_{1} & \text { is } & \mathbb{U}_{n_{1}} & \text { AND } & u_{2} & \text { is } & \mathbb{V}_{n_{2}} & \text { AND } & \cdots & \text { AND } & u_{m} & \text { is } & \mathbb{W}_{n_{m}} & \text { THEN } & y_{R}=z_{R}\end{array}$


In (8a), the firing strengths for each rule are computed, and the vector of firing strengths is normalized in $(8 b)$. The rule conclusions, which are in the form of a linear combination of the input variables augmented by a constant bias $\left(\phi_{i, m+1}\right)$, are given in (8c), and the weighted average-type defuzzifier yielding the crisp output is described in (8d).

$$
\begin{aligned}
w_{i} & =\prod_{j=1}^{m} \mu_{i j}\left(u_{j}\right), \quad i=1,2, \ldots, R, \quad j=1,2, \ldots, m \\
\Omega_{i} & =\frac{w_{i}}{\sum_{k=1}^{R} w_{k}} \\
z_{i} & =\phi_{i, m+1}+\sum_{j=1}^{m} \phi_{i j} u_{j} \\
\tau & =\sum_{i=1}^{R} \Omega_{i} z_{i} .
\end{aligned}
$$

In (8a) and (8c), $u_{j}$ corresponds to the $j$ th entry of the input vector $\mathbf{u}=\left(u_{1}, u_{2}, \ldots, u_{m}\right)$. According to (8d), it is seen that the ANFIS structure has single output. With these in mind, the output of the ANFIS structure can be paraphrased as

$$
\tau=\boldsymbol{\Omega}^{\mathrm{T}} \mathbf{\Phi} \mathbf{V}
$$

where $\Omega:=\left(\Omega_{1}, \Omega_{2}, \ldots, \Omega_{R}\right)^{\mathrm{T}}$ is the vector of normalized firing strengths, $\boldsymbol{\Phi}$ is an $R \times(m+1)$-dimensional matrix containing the adjustable parameters $\left(\phi_{i j}\right)$ of the defuzzifier, and $\mathbf{v}=\left(v_{1}, v_{2}, \ldots, v_{m+1}\right)^{\mathrm{T}}=\left(\begin{array}{ll}\mathbf{u} & 1\end{array}\right)^{\mathrm{T}}$. Our ultimate goal is to adjust the defuzzifier parameters of the ANFIS structure by the fractional tuning scheme such that a desired ANFIS output is obtained.

Although the functional details shown in Fig. 1 are common in most fuzzy models, the specific reasons for choosing the ANFIS structure are as follows. The defuzzification stage of the ANFIS structure prescribes a function [see (8c)] activated at the degree of a firing strength of a rule it belongs to. Every such function that acts as the conclusion of a rule offers significant degrees of freedom in the adjustable parameter space. Although this can be interpreted as an advantage, it constitutes a disadvantage as the initialization of the defuzzifier parameters is more difficult than the simpler fuzzy models. Therefore, an approach utilizing the redundancy of the ANFIS structure without letting the designer be troubled with the initializations is one strong motivation of this paper.

\section{Supervised Training OF ANFIS VIA FRACTIONAL-ORDER ADAPTATION SCHEME}

Given $0<\beta<1$, with zero lower terminal, the RiemannLiouville definition of the $\beta$ th-order fractional derivative operator ${ }_{0} \mathbf{D}_{t}^{\beta}$ is defined by

$$
\begin{aligned}
f^{(\beta)}(t) & ={ }_{0} \mathbf{D}_{t}^{\beta} f(t) \\
& =\frac{1}{\Gamma(1-\beta)} \frac{d}{d t} \int_{0}^{t}(t-\xi)^{-\beta} f(\xi) d \xi
\end{aligned}
$$

where $\Gamma(\cdot)$ is the Gamma function, which is defined as $\Gamma(\beta)=$ $\int_{0}^{\infty} e^{-t} t^{\beta-1} d t$, generalizing the factorial for noninteger arguments. According to this definition, the derivative of a time function $f(t)=t^{\alpha}$, where $\alpha>-1$ and $t \geq 0$, is evaluated as

$$
{ }_{0} \mathbf{D}_{t}^{\beta} t^{\alpha}=\frac{\Gamma(\alpha+1)}{\Gamma(\alpha+1-\beta)} t^{\alpha-\beta} .
$$

Likewise, the Riemann-Liouville definition of the $\beta$ th-order fractional integration operator having zero lower terminal, ${ }_{0} \mathbf{I}_{t}^{\beta}$, is given by

$$
{ }_{0} \mathbf{I}_{t}^{\beta} f(t)=\frac{1}{\Gamma(\beta)} \int_{0}^{t}(t-\xi)^{\beta-1} f(\xi) d \xi .
$$

The material presented in the sequel is based on the aforementioned definitions of fractional differentiation and integration, as well as the following integration rules describing the integral of a derivative $\sigma^{(\beta)}$ and a constant $c$ :

$$
\begin{aligned}
{ }_{0} \mathbf{I}_{t}^{\beta} \sigma^{(\beta)} & =\sigma(t)-\sigma^{(\beta-1)}(0) \frac{t^{\beta-1}}{\Gamma(\beta)} \\
{ }_{0} \mathbf{I}_{t}^{\beta} c & =c \frac{t^{\beta}}{\Gamma(1+\beta)} .
\end{aligned}
$$

An in-depth discussion can be found in [16], [17], and [28]. In the rest of this section, we assume that the target output of the ANFIS structure is known so that the output error is available for parameter tuning process. However, this assumption will be removed in Section V.

Theorem 4.1: Let $\tau$ and $\tau_{d}$ be the output of the ANFIS structure and the target output, respectively. Let the following boundedness conditions hold:

$$
\begin{aligned}
& \left|\sum_{k=1}^{\infty} \sum_{i=1}^{R} \sum_{j=1}^{m+1} \sum_{h=0}^{k}\left(\begin{array}{l}
k \\
h
\end{array}\right)\left(\begin{array}{l}
\beta \\
k
\end{array}\right) \Omega_{i}^{(h)} v_{j}^{(k-h)} \phi_{i j}^{(\beta-k)}\right| \leq \mathcal{B}_{1} \\
& \left|\sum_{k=1}^{\infty} \frac{\Gamma(1+\beta)}{\Gamma(1+k) \Gamma(1-k+\beta)} \sigma^{(k)} \sigma^{(\beta-k)}\right| \leq \mathcal{B}_{2}|\sigma| \\
& \left|\tau_{d}^{(\beta)}\right| \leq \mathcal{B}_{3} .
\end{aligned}
$$

The adaptation law

$$
\boldsymbol{\Phi}^{(\beta)}=-\frac{\boldsymbol{\Omega} \mathbf{v}^{\mathrm{T}}}{\|\mathbf{v}\|^{2}\|\boldsymbol{\Omega}\|^{2}} \mathcal{K} \operatorname{sgn}(\sigma)
$$

with $\sigma:=\tau-\tau_{d}$ being the output error, drives the adjustable parameters of the ANFIS to values such that a hitting in finite time satisfying

$$
\frac{\mathcal{K}-\mathcal{B}_{1}}{\Gamma(1+\beta)} t_{h}^{\beta} \leq \frac{\left|\sigma^{(\beta-1)}(0)\right|+\left|\tau_{d}^{(\beta-1)}(0)\right|}{\Gamma(\beta)} t_{h}^{\beta-1}+\left|\tau_{d}\left(t_{h}\right)\right|
$$

is observed if $\mathcal{K}>\mathcal{B}_{1}+\mathcal{B}_{2}+\mathcal{B}_{3}$ is satisfied.

Proof: Note that observing $\mathbf{s}=\mathbf{0}$ corresponds to the fact that the states of the system are on the sliding manifold. $\sigma=0$, on the other hand, corresponds to the fact that a control signal that eventually results in $\mathbf{s}=\mathbf{0}$ is being produced. The course 
of $\sigma=0$ covers the regime described by $\mathbf{s}=\mathbf{0}$, and therefore, the dynamical conclusions of having $\sigma=0$ are different from $\mathbf{s}=\mathbf{0}$.

Define

$$
\Upsilon:=\sum_{k=1}^{\infty} \sum_{i=1}^{R} \sum_{j=1}^{m+1} \sum_{h=0}^{k}\left(\begin{array}{l}
k \\
h
\end{array}\right)\left(\begin{array}{l}
\beta \\
k
\end{array}\right) \Omega_{i}^{(h)} v_{j}^{(k-h)} \phi_{i j}^{(\beta-k)}
$$

and choose a Lyapunov function candidate $V(t):=\sigma(t)^{2}$. According to the Leibniz rule of fractional differentiation, the $\beta$ th-order time derivative of $V(t)$ can be expanded as follows:

$$
\frac{d^{\beta} V}{d t^{\beta}}=V^{(\beta)}=\sigma^{(\beta)} \sigma+\mathcal{P}
$$

where

$$
\mathcal{P}:=\sum_{k=1}^{\infty} \frac{\Gamma(1+\beta)}{\Gamma(1+k) \Gamma(1-k+\beta)} \sigma^{(k)} \sigma^{(\beta-k)}
$$

Now, check whether the quantity $\sigma^{(\beta)} \sigma$ is negative or not. With these expressions, we have

$$
\begin{aligned}
& \sigma^{(\beta)} \sigma=\left(\tau^{(\beta)}-\tau_{d}^{(\beta)}\right) \sigma \\
& =\left(\left(\boldsymbol{\Omega}^{\mathrm{T}} \mathbf{\Phi} \mathbf{v}\right)^{(\beta)}-\tau_{d}^{(\beta)}\right) \sigma \\
& =\left(\left(\sum_{i=1}^{R} \sum_{j=1}^{m+1} \Omega_{i} v_{j} \phi_{i j}\right)^{(\beta)}-\tau_{d}^{(\beta)}\right) \sigma \\
& =\left(\left(\sum_{k=0}^{\infty} \sum_{i=1}^{R} \sum_{j=1}^{m+1}\left(\begin{array}{l}
\beta \\
k
\end{array}\right)\left(\Omega_{i} v_{j}\right)^{(k)} \phi_{i j}^{(\beta-k)}\right)-\tau_{d}^{(\beta)}\right) \sigma \\
& =\left(\left(\sum_{i=1}^{R} \sum_{j=1}^{m+1}\left(\Omega_{i} v_{j}\right) \phi_{i j}^{(\beta)}\right)\right. \\
& \left.+\left(\sum_{k=1}^{\infty} \sum_{i=1}^{R} \sum_{j=1}^{m+1}\left(\begin{array}{l}
\beta \\
k
\end{array}\right)\left(\Omega_{i} v_{j}\right)^{(k)} \phi_{i j}^{(\beta-k)}\right)-\tau_{d}^{(\beta)}\right) \sigma \\
& =\left(\left(\sum_{i=1}^{R} \sum_{j=1}^{m+1} \Omega_{i} \phi_{i j}^{(\beta)} v_{j}\right)\right. \\
& +\left(\sum_{k=1}^{\infty} \sum_{i=1}^{R} \sum_{j=1}^{m+1} \sum_{h=0}^{k}\left(\begin{array}{l}
k \\
h
\end{array}\right)\left(\begin{array}{l}
\beta \\
k
\end{array}\right)\right. \\
& \left.\left.\times \Omega_{i}^{(h)} v_{j}^{(k-h)} \phi_{i j}^{(\beta-k)}\right)-\tau_{d}^{(\beta)}\right) \sigma \\
& =\left(\boldsymbol{\Omega}^{\mathrm{T}} \boldsymbol{\Phi}^{(\beta)} \mathbf{v}\right) \sigma+\left(\Upsilon-\tau_{d}^{(\beta)}\right) \sigma \\
& =\left(\boldsymbol{\Omega}^{\mathrm{T}}\left(-\frac{\boldsymbol{\Omega} \mathbf{v}^{\mathrm{T}}}{\|\mathbf{v}\|^{2}\|\boldsymbol{\Omega}\|^{2}} \mathcal{K} \operatorname{sgn}(\sigma)\right) \mathbf{v}\right) \sigma+\left(\Upsilon-\tau_{d}^{(\beta)}\right) \sigma \\
& =-\mathcal{K} \operatorname{sgn}(\sigma) \sigma+\left(\Upsilon-\tau_{d}^{(\beta)}\right) \sigma \\
& \leq-\mathcal{K}|\sigma|+|\Upsilon||\sigma|+\left|\tau_{d}^{(\beta)}\right||\sigma| \\
& \leq\left(-\mathcal{K}+\mathcal{B}_{1}+\mathcal{B}_{3}\right)|\sigma| \text {. }
\end{aligned}
$$

The last inequality lets us write

$$
\begin{aligned}
V^{(\beta)} & \leq\left(-\mathcal{K}+\mathcal{B}_{1}+\mathcal{B}_{3}\right)|\sigma|+\mathcal{P} \\
& \leq\left(-\mathcal{K}+\mathcal{B}_{1}+\mathcal{B}_{3}\right)|\sigma|+\mathcal{B}_{2}|\sigma| \\
& =\left(-\mathcal{K}+\mathcal{B}_{1}+\mathcal{B}_{2}+\mathcal{B}_{3}\right)|\sigma| \\
& <0 \text { since } \mathcal{K}>\mathcal{B}_{1}+\mathcal{B}_{2}+\mathcal{B}_{3} .
\end{aligned}
$$

Clearly, $V^{(\beta)}<0$ for $|\sigma|>0$, and this proves that the trajectories in the phase space are attracted by the subspace described by $\sigma=0$.

In its essence, the aforesaid conclusion is to question whether the reaching law characterized by $\sigma^{(\beta)}=-\mathcal{K} \operatorname{sgn}(\sigma)$ forces $\sigma \rightarrow 0$ as time progresses. One could find the answer to this question by writing $\sigma=-\mathcal{K} \mathbf{I}^{\beta} \operatorname{sgn}(\sigma)$ and rearranging this expression by taking the integer-order time derivative as $\dot{\sigma}=-\mathcal{K} \mathbf{I}^{\gamma} \operatorname{sgn}(\sigma)$, where $\gamma=\beta-1$. Under these conditions, it is reported that $\operatorname{sgn}\left(\mathbf{I}^{\gamma} \operatorname{sgn}(\sigma)\right)=\operatorname{sgn}(\sigma)$ if $-1<\gamma<1$ [29]. This result clearly implies that the phase space of a sliding-mode control system enforcing the reaching law $\sigma^{(\beta)}=$ $-\mathcal{K} \operatorname{sgn}(\sigma)$ has an attractor characterized by $\sigma=0$ as $\sigma \dot{\sigma}<0$ for $\sigma \neq 0$.

Now, we must prove that first hitting to the switching function occurs in finite time, which is denoted by $t_{h}$. Evaluate $\sigma^{(\beta)}$ utilizing (18) as given next

$$
\sigma^{(\beta)}=-\mathcal{K} \operatorname{sgn}(\sigma)+\Upsilon-\tau_{d}^{(\beta)} .
$$

Applying the fractional integration operator described in (12) to both sides of (23) and considering the particular result in (13) with final time $t=t_{h}$, one gets

$$
\begin{aligned}
\sigma\left(t_{h}\right)-\sigma^{(\beta-1)} & (0) \frac{t_{h}^{\beta-1}}{\Gamma(\beta)} \\
& =\frac{-\mathcal{K} \operatorname{sgn}(\sigma(0))}{\Gamma(1+\beta)} t_{h}^{\beta}+{ }_{0} \mathbf{I}_{t_{h}}^{\beta}\left(\Upsilon-\tau_{d}^{(\beta)}\right) .
\end{aligned}
$$

Noting that $\sigma(t)=0$ at $t=t_{h}$, multiplying both sides of (24) by $\operatorname{sgn}(\sigma(0))$, we have

$$
\begin{aligned}
& -\sigma^{(\beta-1)}(0) \operatorname{sgn}(\sigma(0)) \frac{t_{h}^{\beta-1}}{\Gamma(\beta)}=\frac{-\mathcal{K}}{\Gamma(1+\beta)} t_{h}^{\beta} \\
& +{ }_{0} \mathbf{I}_{t_{h}}^{\beta}(\operatorname{sgn}(\sigma(0)) \Upsilon)-{ }_{0} \mathbf{I}_{t_{h}}^{\beta}\left(\operatorname{sgn}(\sigma(0)) \tau_{d}^{(\beta)}\right) .
\end{aligned}
$$

Due to the definition given in (12) and the result in (14), we have

$$
\begin{aligned}
{ }_{0} \mathbf{I}_{t_{h}}^{\beta}(\operatorname{sgn}(\sigma(0)) \Upsilon) & \leq{ }_{0} \mathbf{I}_{t_{h}}^{\beta}|\Upsilon| \\
& \leq{ }_{0} \mathbf{I}_{t_{h}}^{\beta} \mathcal{B}_{1} \\
& =\mathcal{B}_{1} \frac{t_{h}^{\beta}}{\Gamma(1+\beta)} .
\end{aligned}
$$

Similarly

$$
\begin{aligned}
{ }_{0} \mathbf{I}_{t_{h}}^{\beta}\left(\operatorname{sgn}(\sigma(0)) \tau_{d}^{(\beta)}\right) & =\operatorname{sgn}(\sigma(0))_{0} \mathbf{I}_{t_{h}}^{\beta} \tau_{d}^{(\beta)} \\
& =\operatorname{sgn}(\sigma(0))\left(\tau_{d}\left(t_{h}\right)-\tau_{d}^{(\beta-1)}(0) \frac{t_{h}^{\beta-1}}{\Gamma(\beta)}\right) .
\end{aligned}
$$


Substituting the results in (26) and (27) into (25), we obtain an inequality given as

$$
\begin{aligned}
-\sigma^{(\beta-1)}(0) \operatorname{sgn}(\sigma(0)) \frac{t_{h}^{\beta-1}}{\Gamma(\beta)} \leq & \frac{-\mathcal{K}}{\Gamma(1+\beta)} t_{h}^{\beta}+\mathcal{B}_{1} \frac{t_{h}^{\beta}}{\Gamma(1+\beta)} \\
& -\operatorname{sgn}(\sigma(0)) \tau_{d}\left(t_{h}\right) \\
& +\tau_{d}^{(\beta-1)}(0) \operatorname{sgn}(\sigma(0)) \frac{t_{h}^{\beta-1}}{\Gamma(\beta)}
\end{aligned}
$$

Straightforward manipulations will lead to the inequality in (19). Clearly, the left-hand side of the inequality in (19) is a nonconverging and monotonically increasing function of $t_{h}$. On the other hand, the right-hand side of the inequality is a monotonically decreasing function of $t_{h}$. Looking at the powers of the arguments, one sees that an intersection is inevitable. With these facts, the inequality is satisfied on the interval $t_{h} \in(0, \alpha]$, where $\alpha$ is the point of intersection of the two expressions lying on the left- and right-hand sides of (19). According to this discussion, one can see that $t_{h} \leq \alpha$, and particularly for $\beta=0.5$, we have the following value:

$\alpha=\left(\frac{\left|\tau_{d}\left(t_{h}\right)\right|+\sqrt{\left|\tau_{d}\left(t_{h}\right)\right|^{2}+4 \frac{\mathcal{K}-\mathcal{B}_{1}}{\Gamma(1+\beta)} \frac{\left|\sigma^{(\beta-1)}(0)\right|+\left|\tau_{d}^{(\beta-1)}(0)\right|}{\Gamma(\beta)}}}{2 \frac{\mathcal{K}-\mathcal{B}_{1}}{\Gamma(1+\beta)}}\right)^{2}$.

Now, we turn our attention to the assumptions we made in (15)-(17). Obviously, the assumptions are rather stringent. The control system presented here would be globally stable if these conditions hold true for the entire course of operation; however, imposing such bounds makes the presented design valid only within a local region. In the sequel, we present an example to demonstrate that the mentioned local region is practically large enough to observe a highly satisfactory performance.

\section{Conditions For Obtaining $\operatorname{sgn}(\sigma)$}

In Section II, we summarize the conventional sliding-mode control scheme for multi-input-multi-output systems of the form (1). On the other hand, if we could know a supervisory signal to compute $\sigma$, we would use it directly in the fractional adaptation scheme given in (18). However, the nature of the control systems does not provide such information; instead, one has to develop strategies to observe a desired response in the closed loop by utilizing available quantities. Therefore, a critically important stage of the approach presented in this paper is to extract an equivalent measure about the sign of the error on the control signal to use in the parameter tuning scheme. In other words, we need to develop a strategy, together with a set of assumptions, such that our tuning scheme drives the closed-loop system toward the behavior that can be obtained via the conventional sliding-mode controller without knowing the system parameters.
For this purpose, denote the response of the ANFIS controllers by $\boldsymbol{\tau}_{A}$, which is $n \times 1$, and set $\mathbf{T}:=\tau_{A}$. Because there are $n$ subsystems, there are $n$ ANFIS controllers. Consider the difference

$$
\begin{aligned}
\sigma & =\boldsymbol{\tau}_{\mathbf{A}}-\boldsymbol{\tau}_{\mathbf{S M C}} \\
& =\boldsymbol{\tau}_{\mathbf{A}}+(\boldsymbol{\Lambda} G(\boldsymbol{\Theta}))^{-1} \boldsymbol{\Lambda}\left(F(\boldsymbol{\Theta})-\dot{\boldsymbol{\Theta}}_{d}\right)+(\boldsymbol{\Lambda} G(\boldsymbol{\Theta}))^{-1} \mathbf{Q} \operatorname{sgn}(\mathbf{s}) \\
& =\mathbf{J} \operatorname{sgn}(\mathbf{s})+\mathbf{H}
\end{aligned}
$$

where $\mathbf{J}:=(\boldsymbol{\Lambda} G(\boldsymbol{\Theta}))^{-1} \mathbf{Q} \quad$ and $\quad \mathbf{H}:=\boldsymbol{\tau}_{A}+(\boldsymbol{\Lambda} G(\boldsymbol{\Theta}))^{-1}$ $\boldsymbol{\Lambda}\left(F(\boldsymbol{\Theta})-\dot{\boldsymbol{\Theta}}_{d}\right)$. Let $\tilde{\mathbf{J}}$ be a diagonal matrix where $\tilde{\mathbf{J}}_{i i}=\mathbf{J}_{i i}$. Let $\tilde{\mathbf{H}}:=\mathbf{H}+(\mathbf{J}-\tilde{\mathbf{J}}) \operatorname{sgn}(\mathbf{s})$. With these definitions, (30) can be paraphrased as

$$
\boldsymbol{\sigma}=\tilde{\mathbf{J}} \operatorname{sgn}(\mathbf{s})+\tilde{\mathbf{H}}
$$

whose rows can explicitly be written as

$$
\sigma_{i}=\tilde{\mathbf{J}}_{i i} \operatorname{sgn}\left(s_{i}\right)+\tilde{\mathbf{H}}_{i}, \quad i=1,2, \ldots, n .
$$

The expression in (32) stipulates that if $\left|\tilde{\mathbf{H}}_{i}\right|<\tilde{\mathbf{J}}_{i i}$, then $\operatorname{sgn}\left(\sigma_{i}\right)=\operatorname{sgn}\left(s_{i}\right)$. In other words, aside from the three bound conditions given $[(15)-(17)]$, a fourth one is given as follows:

$$
0 \leq\left|\tilde{\mathbf{H}}_{i}\right|<\tilde{\mathbf{J}}_{i i}, \quad i=1,2, \ldots, n
$$

Note that one can obtain infinitely many different designs of $\mathbf{J}$, including those satisfying the set of inequalities earlier. Aside from the components coming from the system dynamics and the desired response, this depends also upon $\boldsymbol{\Lambda}$ and $\mathrm{Q}$, the choice of which can change the desired properties of the sliding mode as well as the reaching mode. Therefore, one needs to check whether $\tilde{\mathbf{J}}_{i i}$ is positive or not.

Corollary: If the inequalities in (15)-(17), and (33) are satisfied, the tuning law in (18) enforces reaching $\sigma_{i}=0$ for $\forall i$, and this triggers the emergence of the sliding mode in the traditional sense. However, the conditions derived in this section imply a class of plants where such an induction could be valid. In the next section, we give the dynamical description of a 2-DOF robot.

\section{DyNAMics of THE Robot ARM AND THE CONTROL PROBLEM}

In this paper, we consider the following system to visualize the contributions of this paper. The motivation for choosing this system is the nonlinear and coupled nature of differential equations describing the behavior. Furthermore, the adverse effects of noise, large initial conditions, and varying payload conditions make the control problem a challenge for conventional approaches. The dynamics of the robot is given by

$$
M(\theta) \ddot{\theta}+C(\theta, \dot{\theta})=\tau-\eta
$$


where $\theta=\left(\begin{array}{ll}\theta_{1} & \theta_{2}\end{array}\right)^{\mathrm{T}}$ is the vector of angular positions in radians and $\dot{\theta}=\left(\begin{array}{ll}\dot{\theta}_{1} & \dot{\theta}_{2}\end{array}\right)^{\mathrm{T}}$ is the vector of angular velocities in radians per second. In (34), $\tau=\left(\begin{array}{ll}\tau_{1} & \tau_{2}\end{array}\right)^{\mathrm{T}}$ is the vector of control inputs (torques), and $\eta=\left(\begin{array}{ll}\eta_{1} & \eta_{2}\end{array}\right)^{\mathrm{T}}$ is the vector of friction forces. The terms in (34) are given as follows:

$$
\begin{aligned}
M(\theta) & =\left(\begin{array}{cc}
p_{1}+2 p_{3} \cos \left(\theta_{2}\right) & p_{2}+p_{3} \cos \left(\theta_{2}\right) \\
p_{2}+p_{3} \cos \left(\theta_{2}\right) & p_{2}
\end{array}\right) \\
C(\theta, \dot{\theta}) & =\left(\begin{array}{c}
-\dot{\theta}_{2}\left(2 \dot{\theta}_{1}+\dot{\theta}_{2}\right) p_{3} \sin \theta_{2} \\
\dot{\theta}_{1}^{2} p_{3} \sin \theta_{2}
\end{array}\right)
\end{aligned}
$$

where $p_{1}=3.31655+0.18648 M_{p}, p_{2}=0.1168+0.0576 M_{p}$, and $p_{3}=0.16295+0.08616 M_{p}$. Here, $M_{p}$ denotes the payload mass. The details of the plant model can be found in [30] and [31]. The constraints regarding the plant dynamics are $\left|\tau_{1}\right| \leq 245 \mathrm{~N}$ and $\left|\tau_{2}\right| \leq 39.2 \mathrm{~N}$, and the friction terms are $\eta_{1}=4.9 \operatorname{sgn}\left(\dot{\theta}_{1}\right)$ and $\eta_{2}=1.67 \operatorname{sgn}\left(\dot{\theta}_{2}\right)$.

The control problem is to force the system states to predefined and differentiable trajectories within the workspace of the robot. More explicitly, $e_{1}=\theta_{1}-\theta_{d, 1}$ and $e_{2}=\theta_{2}-\theta_{d, 2}$ and the first-order (integer) time derivatives of these error terms are desired to converge the origin of the phase space.

According to the presented analysis and the previous model, the dynamics of the system under control can be cast into the representation $\dot{\boldsymbol{\Theta}}=F(\boldsymbol{\Theta})+G(\boldsymbol{\Theta}) \mathbf{T}$, and we have $(\boldsymbol{\Lambda} G)^{-1}=$ $M(\theta)$. More explicitly

$$
\begin{aligned}
(\boldsymbol{\Lambda} G)^{-1} \mathbf{Q}= & \left(\begin{array}{c}
\mathbf{Q}_{11}\left(p_{1}+2 p_{3} \cos \left(\theta_{2}\right)\right) \operatorname{sgn}\left(s_{1}\right) \\
\mathbf{Q}_{22} p_{3} \operatorname{sgn}\left(s_{2}\right)
\end{array}\right) \\
& +\left(\begin{array}{c}
\mathbf{Q}_{22}\left(p_{1}+p_{3} \cos \left(\theta_{2}\right)\right) \operatorname{sgn}\left(s_{2}\right) \\
\mathbf{Q}_{11}\left(p_{1}+p_{3} \cos \left(\theta_{2}\right)\right) \operatorname{sgn}\left(s_{1}\right)
\end{array}\right)
\end{aligned}
$$

The aforementioned separation of terms suggests that $\tilde{\mathbf{J}}_{11}=$ $\mathbf{Q}_{11}\left(p_{1}+2 p_{3} \cos \left(\theta_{2}\right)\right)>0$ and $\tilde{\mathbf{J}}_{22}=\mathbf{Q}_{22} p_{3}>0$ for every possible angular state and payload condition. Clearly, the devised approach is suitable for mechanical systems, robots, and systems as they have a positive definite inertia matrix. In the next section, we present the simulation studies comparatively with the integer-order integration scheme in the parameter adaptation stage.

\section{Simulation Results}

The presented approach is implemented for the plant introduced in Section VI. The block diagram of the control system is shown in Fig. 3, where it is seen that the state of the system under control is measurable and that one ANFIS controller is dedicated to produce each component of the control signal $\mathbf{T}$.

The membership functions of both ANFIS controllers are chosen as shown in Fig. 4. This selection is made after a few trials. Angular positions and velocities are measured, and the controllers receive the relevant error in position and velocity for each link. Three linguistic labels, namely, Negative, Zero, and Positive, are chosen, and a total of $R=9$ rules are contained in

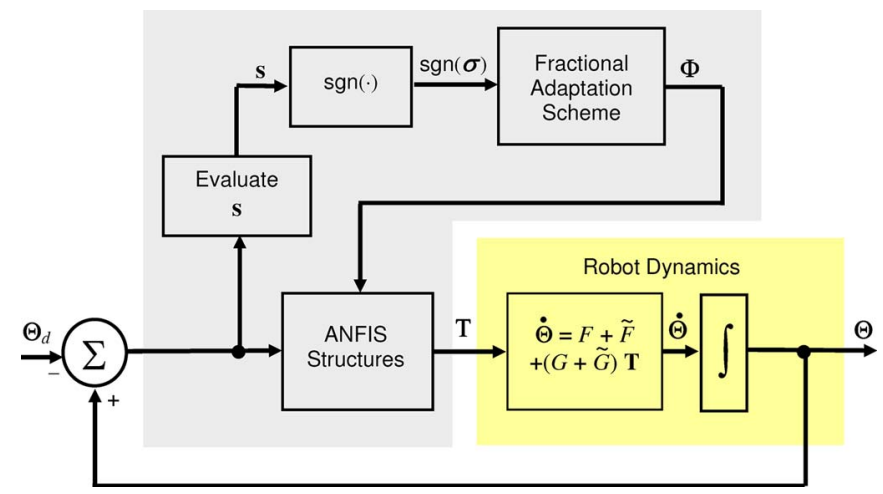

Fig. 3. Block diagram of the control system.

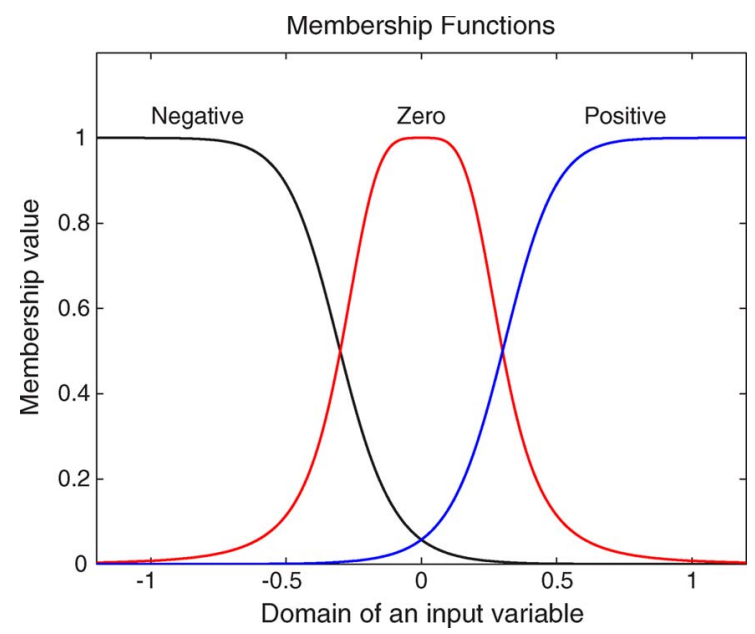

Fig. 4. Membership functions utilized in both ANFIS structures.

the rule base. It is important to note that the membership functions cover the entire space and that there is no subregion over which a conclusion is not defined. This is critically important; otherwise, $\|\boldsymbol{\Omega}\|^{2}$ may approach zero, and this would lead to unnecessarily large tuning effort causing possible instabilities. One may define more linguistic labels than what we choose and obtain a better linguistic resolution for better performance. Clearly, the need for utilizing finer rule resolution depends upon the requirements of the problem in hand.

The right-hand side of (18) is computed, and the fractional integration is achieved through the approximation called CRONE, a French acronym for Commande robuste d'ordre non-entier meaning robust fractional-order control. A frequency range is set first, and a set of zeros and poles is organized in such a way that the desired order of differintegration is approximated as follows:

$$
s^{-\beta} \approx \prod_{i=1}^{N} \frac{1+\frac{s}{\omega_{z_{i}}}}{1+\frac{s}{\omega_{p_{i}}}}
$$

where $s$ denotes the Laplace transform variable. In the previous, $\omega_{z_{i}}$ and $\omega_{p_{i}}$ are adjusted automatically to yield the best match. The interested reader is referred to [32] for the algorithm scheduling these parameters. We choose $N=25$ for obtaining a good match, and $(0.01,100) \mathrm{rad} / \mathrm{s}$ as the frequency range over which the approximation will be valid. The control system 

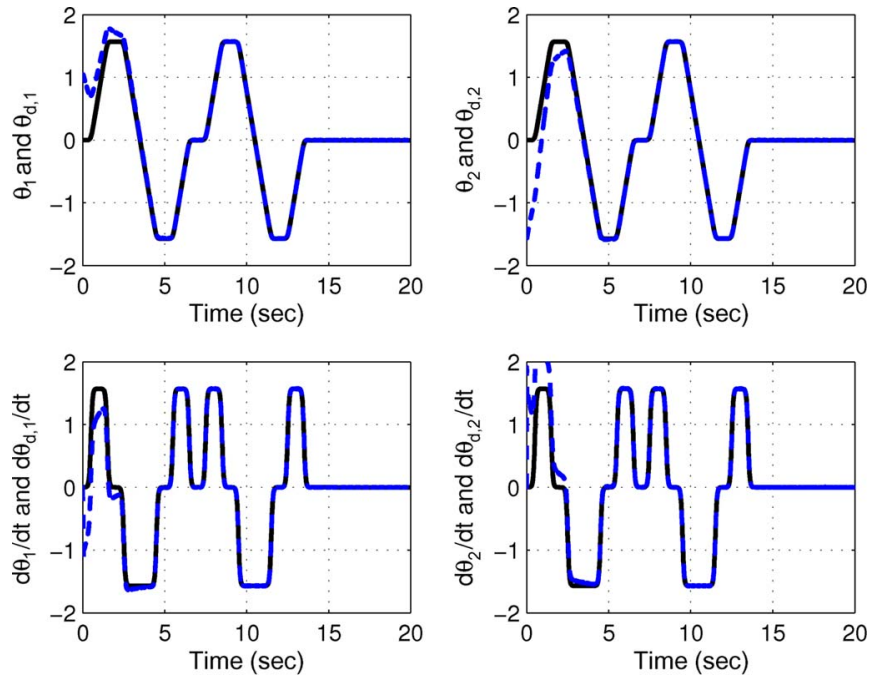

Fig. 5. Reference trajectories and the response of the robot.

runs for 20 s, and the reference trajectories shown in Fig. 5 are used. The solid curves represent the reference trajectories, whereas the dashed ones stand for the response of the robot. During the operation, a 5 -kg payload is grasped when $t=2 \mathrm{~s}$ and is released when $t=5 \mathrm{~s}$, and this is repeated when the robot is motionless at $t=9$ and $12 \mathrm{~s}$. The manipulator is desired to stay motionless after $t=15 \mathrm{~s}$.

It should be noted that the payload scenario is a significant disturbance changing the dynamics of the plant suddenly. Another difficulty is the initial conditions that the ANFIS controllers are supposed to alleviate. Initially, $\theta_{d, 1}=\theta_{d, 2}=0$, the system is motionless, and $\theta_{1}(0)=\pi / 3$ and $\theta_{2}(0)=-\pi / 2$, which are large enough to test the performance of a control scheme. The simulations are carried out with a time step of $2.5 \mathrm{~ms}$, and $\mathcal{K}_{1}=200$ and $\mathcal{K}_{2}=100$ values are chosen after just a few trials. The sliding lines for both links are set by choosing $\lambda_{i}=1$, where $i=1,2$. Other than these, in order to avoid exciting any undesired chattering phenomenon associated tightly with the discontinuous nature of the sign function, we choose $\operatorname{sgn}(\sigma) \approx(\sigma /|\sigma|+\delta)$, with $\delta$ being the parameter determining the slope around the origin. This paper considers $\delta=0.01$ introducing a very thin boundary layer.

The discrepancies between the reference trajectories and the system response are shown in Fig. 6, where an exponential convergence is apparent even in the presence of noise corrupting the observed system states and the changes in the system dynamics due to the payload variations.

The behavior in the phase space shown in Fig. 7 is another evidence of robustness of the control system and insensitivity to variations in the plant dynamics.

In Fig. 8, the applied control signals are given with the window graphs for better visualization of the initial transients. Due to the large initial positional errors, the control efforts during the first $100 \mathrm{~ms}$ have higher magnitudes than what comes later. The effect of noisy observations on the control signal is another conclusion that is worth mentioning.

A sum-squared value for the controller parameters, which are all started from zero, are shown separately in Fig. 9, where it is clearly visible that the norm values evolve bounded and settle
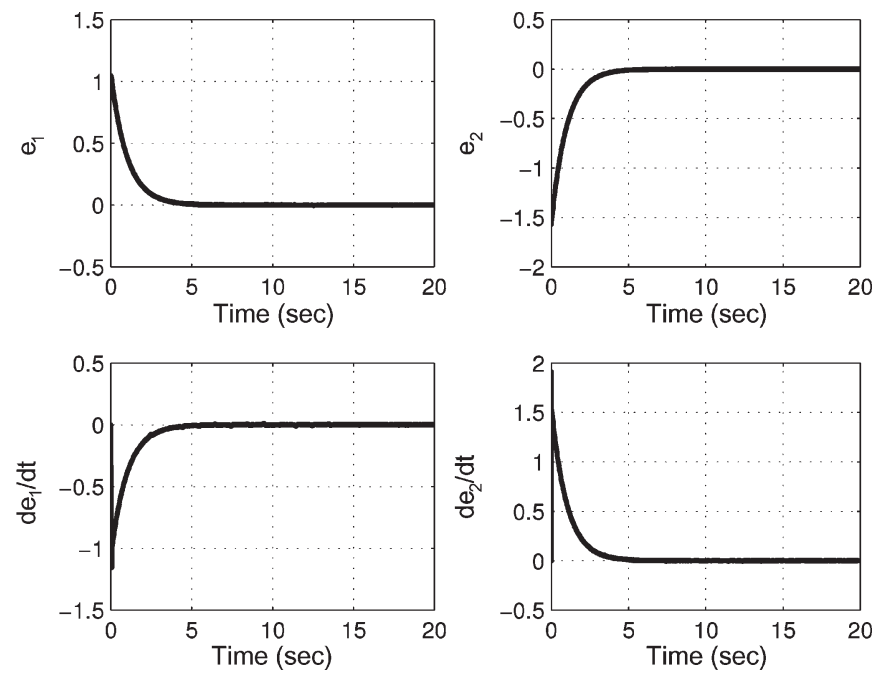

Fig. 6. State tracking errors.
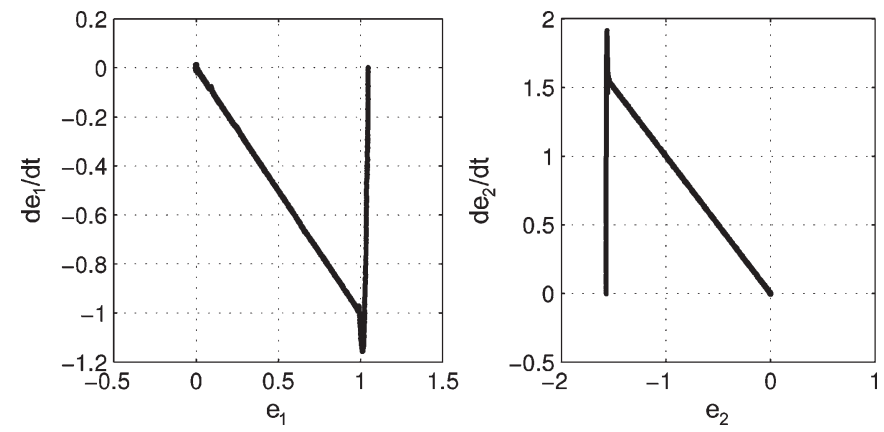

Fig. 7. Behavior in the phase space.
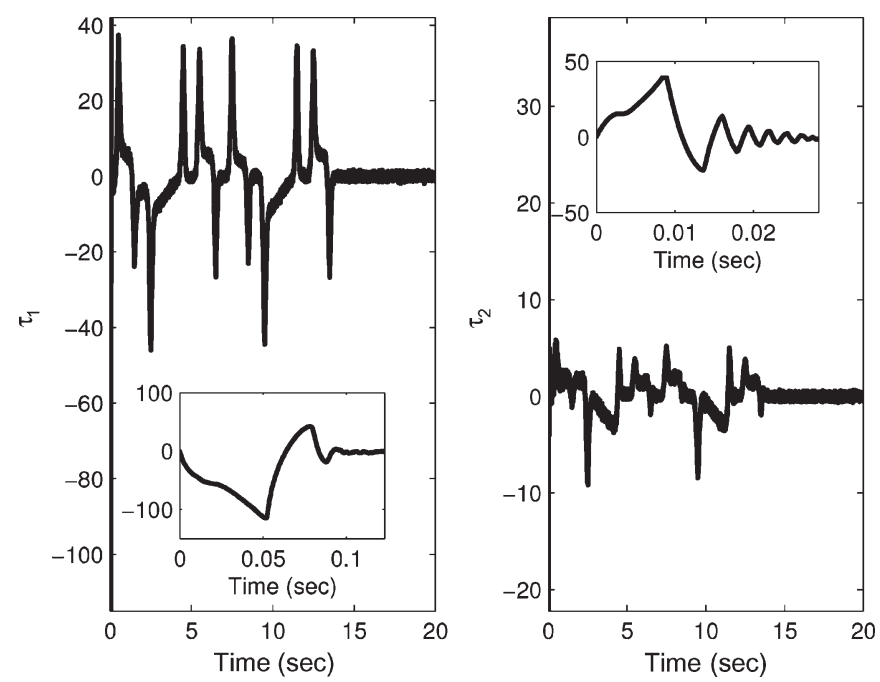

Fig. 8. Applied control signals and their initial transients.

down to constant values. If we remember the reference profiles, the system is desired to be motionless after $t>15 \mathrm{~s}$. This means that the tuning activity during this time is subject to the effects of noise. That is to say, the system is at a desired state, but we would like to figure out how the parameter tuning mechanism functions during this period. According to Fig. 9, it is seen that the value of $\operatorname{trace}\left(\boldsymbol{\Phi}^{\mathrm{T}} \boldsymbol{\Phi}\right)$ for the two ANFIS controllers stay within constant values. 

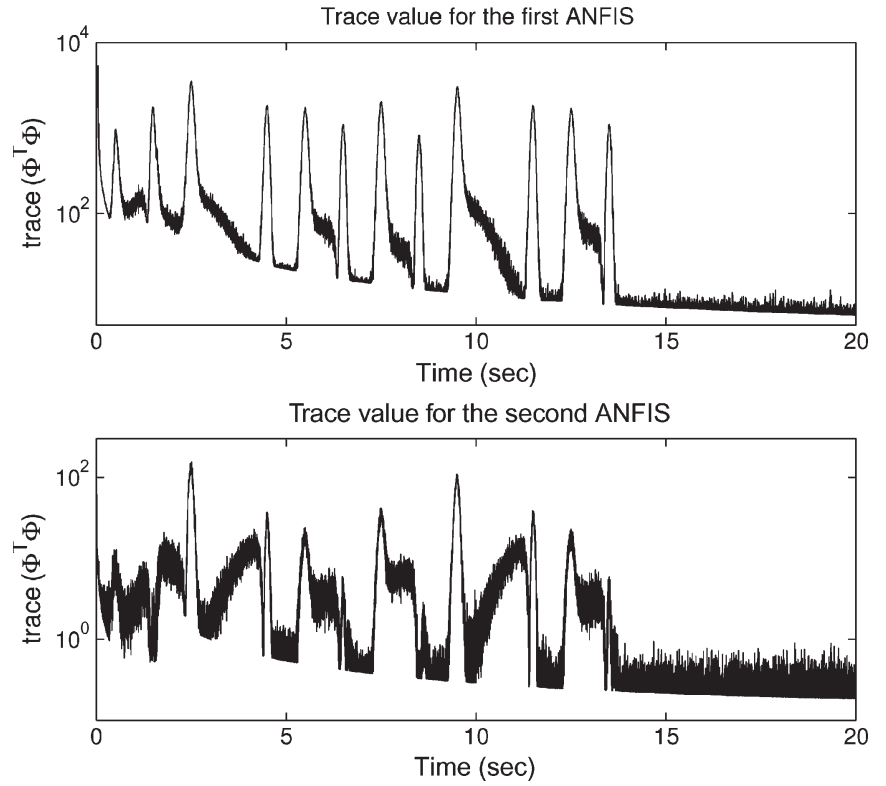

Fig. 9. Time evolution of the controller parameters for base link (top) and elbow link (bottom).
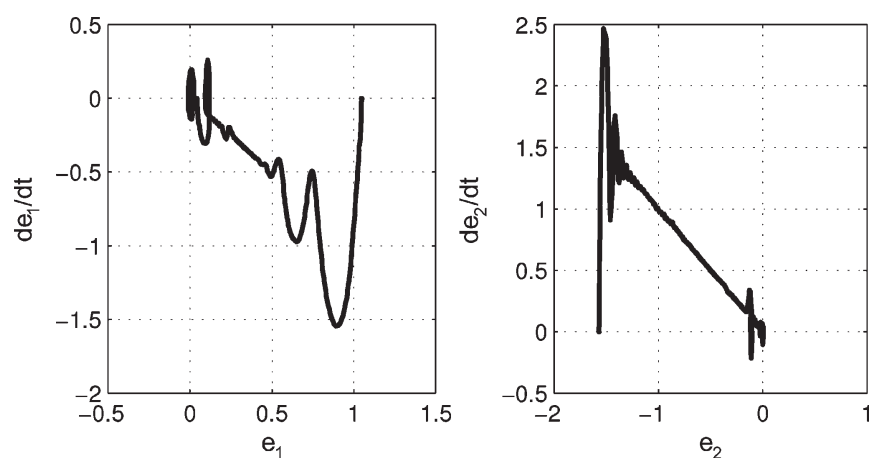

Fig. 10. Behavior in the phase space for the integer case, i.e., $\beta=1$.

In Fig. 10, we demonstrate the results obtained with integerorder version of the same tuning scheme. In this case, several hits occur before the error vector gets trapped to the sliding manifold, and we see the adverse effects of the changing payload conditions as slight temporary deviations from the switching manifold. Clearly, a comparison of the cases $\beta=1$ and $\beta=0.5$ under the identical operating conditions suggests the use of the fractional-order form of the tuning law as it creates a more smooth sliding motion than the integer-order one.

As a last issue, we focus on the time evolution of the variables seen in (33) (Fig. 11). For $\mathbf{Q}=\operatorname{diag}[100$ 500], the system is simulated under the same initial conditions but with the controller described in (6), i.e., with $\tau_{\text {SMC }}$. Clearly, the conditions in (33) are satisfied for the chosen $\mathbf{Q}$. Indeed, one may choose many different $\mathbf{Q}$ matrices satisfying the conditions in (33).

\section{CONCLUSION}

This paper discusses a fractional integration scheme for FSMC. Due to its popularity and parametric redundancy, the ANFIS structure is used as the controller, and it is shown that the approach is applicable to multi-input-multi-output systems.
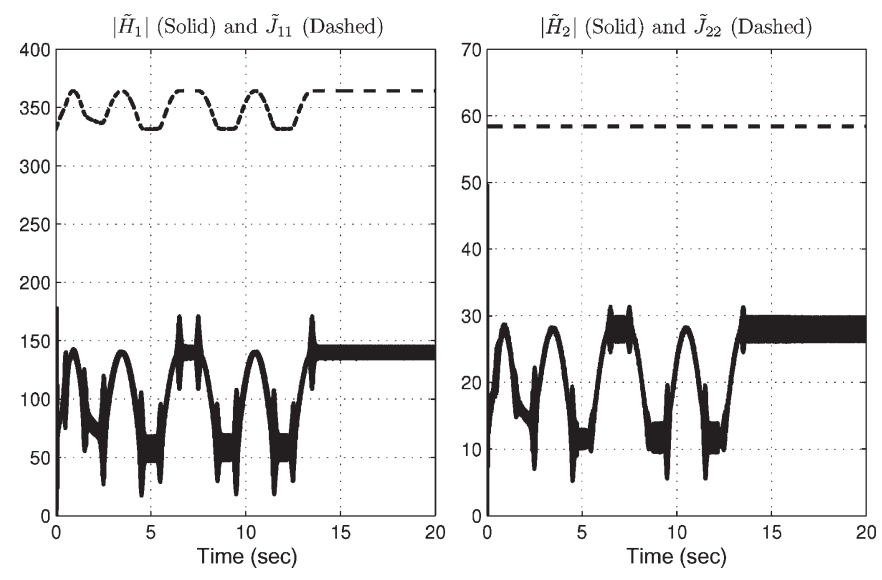

Fig. 11. Time evolution of the variables involved in (33).

The crux of the approach is the use of sign equality in between the switching function defined for one subsystem and the error on the relevant control signal. Because the latter is not known due to the nature of the control problems, this paper demonstrates the conditions under which one can mention such equalities. For the fractional adaptation scheme, this paper provides an upper bound for the hitting time, and parallel to the claims, in the application example, it is shown that the presented form of the adaptation law provides, compared to its integerorder counterpart, which is only computationally simple, the following:

1) better tracking capabilities;

2) better robustness and disturbance rejection capabilities;

3) better exploitation of the parametric redundancy provided by ANFIS;

4) removal of the difficulty in assigning the initial values to the defuzzifier parameters.

Briefly, according to the presented results, the fractional-order tuning law outperforms the tuning mechanisms exploiting integer-order operators.

By demonstrating the usefulness of fractional-order operators in adaptation mechanisms, this paper addresses a wide range of applications from the field of adaptive control; more specifically, the field of adaptive sliding-mode control is focused in this paper. Future work of the author aims to provide a rigorous proof for bounded evolution of the adjustable parameters.

\section{ACKNOWLEDGMENT}

The author would like to thank Dr. D. Valério for his efforts in developing the Matlab toolbox Ninteger v.2.3 (found at http://mega.ist.utl.pt/dmov/ninteger/ninteger.htm) used in this paper.

\section{REFERENCES}

[1] K. J. Åström and B. Wittenmark, Adaptive Control, 2nd ed. Reading, MA: Addison-Wesley, 1995.

[2] K. S. Narendra and K. Parthasarathy, "Identification and control of dynamical systems using neural networks," IEEE Trans. Neural Netw., vol. 1, no. 1, pp. 4-27, Mar. 1990.

[3] L. X. Wang, Adaptive Fuzzy Systems and Control, Design and Stability Analysis. Englewood Cliffs, NJ: Prentice-Hall, 1994. 
[4] J.-S. R. Jang, C.-T. Sun, and E. Mizutani, Neuro-Fuzzy and Soft Computing. Englewood Cliffs, NJ: Prentice-Hall, 1997.

[5] S. Haykin, Neural Networks. New York: Macmillan, 1994.

[6] R. Palm, "Robust-control by fuzzy sliding mode," Automatica, vol. 30, no. 9, pp. 1429-1437, Sep. 1994.

[7] C. Bonivento, C. Fantuzzi, and L. Martini, "Adaptive fuzzy logic controller synthesis via a sliding mode approach," in Proc. 3rd Eur. Control Conf., Rome, Italy, Sep. 5-8, 1995.

[8] K. Erbatur, O. Kaynak, A. Sabanovic, and I. Rudas, "Fuzzy adaptive sliding mode control of a direct drive robot," Robot. Auton. Syst., vol. 19, no. 22, pp. 215-227, 1996.

[9] R. G. Berstecher, R. Palm, and H. D. Unbehauen, "An adaptive fuzzy sliding-mode controller," IEEE Trans. Ind. Electron., vol. 48, no. 1, pp. 18-31, Feb. 2001.

[10] S. C. Tong and H. X. Li, "Fuzzy adaptive sliding-mode control for MIMO nonlinear systems," IEEE Trans. Fuzzy Syst., vol. 11, no. 3, pp. 354-360, Jun. 2003.

[11] B. A. Bazzi and N. G. Chalhoub, "Fuzzy sliding mode controller for a flexible single-link robotic manipulator," J. Vib. Control, vol. 11, no. 2, pp. 295-314, 2005.

[12] O. Kaynak, K. Erbatur, and M. Ertugrul, "The fusion of computationally intelligent methodologies and sliding-mode control-A survey," IEEE Trans. Ind. Electron., vol. 48, no. 1, pp. 4-17, Feb. 2001.

[13] H. F. Ho, Y. K. Wong, and A. B. Rad, "Robust fuzzy tracking control for robotic manipulators," Simul. Model. Pract. Theory, vol. 15, no. 7, pp. 801-816, 2007.

[14] H. T. Yau, "Chaos synchronization of two uncertain chaotic nonlinear gyros using fuzzy sliding mode control," Mech. Syst. Signal Process., vol. 22, no. 2, pp. 408-418, 2008.

[15] Z. Z. Chen, C. H. Shan, and H. L. Zhu, "Adaptive fuzzy sliding mode control algorithm for a non-affine nonlinear system," IEEE Trans. Ind. Inf., vol. 3, no. 4, pp. 302-311, Nov. 2007.

[16] K. B. Oldham and J. Spanier, The Fractional Calculus. New York: Academic, 1974.

[17] I. Podlubny, Fractional Differential Equations, 1st ed. New York: Elsevier, 1998.

[18] D. Matignon, "Stability properties for generalized fractional differential systems," in Proc. ESAIM, 1998, vol. 5, pp. 145-158.

[19] S. Momani and S. Hadid, "Lyapunov stability solutions of fractional integrodifferential equations," Int. J. Math. Math. Sci., vol. 2004, no. 47, pp. 2503-2507, 2004.

[20] B. M. Vinagre, I. Petraš, I. Podlubny, and Y. Q. Chen, "Using fractional order adjustment rules and fractional order reference models in modelreference adaptive control," Nonlinear Dyn., vol. 29, no. 1-4, pp. 269279, Jul. 2002.

[21] S. Ladaci and A. Charef, "On fractional adaptive control," Nonlinear Dyn., vol. 43, no. 4, pp. 365-378, Mar. 2006.

[22] V. I. Utkin, Sliding Modes in Control Optimization. New York: SpringerVerlag, 1992.

[23] C. Edwards and S. K. Spurgeon, Sliding Mode Control Theory and Applications. New York: Taylor \& Francis, 1998
[24] W. Perruquetti and J. P. Barbot, Sliding Mode Control in Engineering. New York: Marcel Dekker, 2002

[25] Z. Hou, Q. Shen, and H. Li, "Nonlinear system identification based on ANFIS," in Proc. Int. Conf. Neural Netw. Signal Process., Beijing, China, Dec. $14-17,2003$, pp. $510-512$.

[26] S. Refaat and S. Nahavandi, "Nonlinear identification of pneumatic servodrive," Int. J. Model. Simul., vol. 26, no. 1, pp. 11-16, Jan. 2006.

[27] T. Takagi and M. Sugeno, "Fuzzy identification of systems and its applications to modeling and control," IEEE Trans. Syst., Man, Cybern., vol. SMC-15, no. 1, pp. 116-132, Jan. 1985.

[28] S. Das, Functional Fractional Calculus for System Identification and Controls, 1st ed. Berlin, Germany: Springer-Verlag, 2008.

[29] B. M. Vinagre and A. J. Calderón, "On fractional sliding mode control," in Proc.7th CONTROLO, Lisbon, Portugal, Sep. 11-13, 2006.

[30] Direct Drive Manipulator $R \& D$ Package User Guide, Integrated Motions Inc., Berkeley, CA, 1992

[31] M. Ö. Efe and O. Kaynak, "A comparative study of soft computing methodologies in identification of robotic manipulators," Robot. Auton. Syst., vol. 30, no. 3, pp. 221-230, Feb. 2000.

[32] D. Valèrio, Ninteger v.2.3 Fractional Control Toolbox for MatLab, 2005.

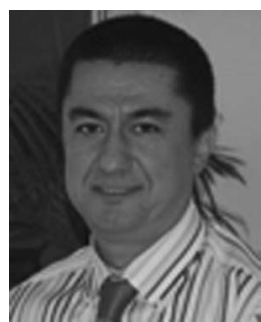

Mehmet Önder Efe (M'04-SM'07) was born in Turkey, 1972. He received the B.Sc. degree from the Istanbul Technical University, Istanbul, Turkey, in 1993, and the M.S. and Ph.D. degree from Bogaziçi University, Istanbul, Turkey, in 1996 and 2000.

Between August 1996 and December 2000, he was with the Mechatronics Research and Application Center, Bogaziçi University, as a Research Assistant. During 2001, he was a Postdoctoral Research Fellow at the Electrical and Computer Engineering Department (AML and ICES), Carnegie Mellon University, Pittsburgh, PA. Between January 2002 and July 2003, he was with the Electrical Engineering Department, The Ohio State University, Columbus, as a Postdoctoral Research Associate. He was with the Collaborative Center of Control Science. During September 2003-June 2004, he was with the Department of Mechatronics Engineering, Atilim University, Ankara, Turkey. He was promoted to the Associate Professor position in April 2004. Since August 2004, he has been with the Department of Electrical and Electronics Engineering, TOBB Economics and Technology University, Ankara, Turkey. He was the Head of the department between August 2004 and July 2007, and he has been chairing the department since June 2008. He is the author or coauthor of more than 100 technical publications focusing on the applications of computational intelligence and systems and control theory.

Dr. Efe has been the Head of the IEEE CSS Turkey Chapter since January 2007. He serves as an Associate Editor for the Transactions of the Institute of Measurement and Control, the International Journal of Industrial Electronics and Control, and Advances in Fuzzy Systems. 